\title{
Accelerated Simulation Study of Space Charge Effects in Quadrupole lon Traps Using GPU Techniques
}

\author{
Xingchuang Xiong, ${ }^{1,2}$ Wei Xu, ${ }^{1,3}$ Xiang Fang, ${ }^{2}$ Yulin Deng, ${ }^{1}$ Zheng Ouyang ${ }^{3,4}$ \\ ${ }^{1}$ School of Life Science, Beijing Institute of Technology, Beijing 100081, China \\ ${ }^{2}$ National Institute of Metrology, Beijing 100013, China \\ ${ }^{3}$ Weldon School of Biomedical Engineering, Purdue University, West Lafayette, IN 47907, USA \\ ${ }^{4}$ Center for Analytical Instrumentation Development, Purdue University, West Lafayette, IN 47907, USA
}

\begin{abstract}
Space charge effects play important roles in the performance of various types of mass analyzers. Simulation of space charge effects is often limited by the computation capability. In this study, we evaluate the method of using graphics processing unit (GPU) to accelerate ion trajectory simulation. Simulation using GPU has been compared with multi-core central processing unit (CPU), and an acceleration of about 390 times have been obtained using a single computer for simulation of up to $10^{5}$ ions in quadrupole ion traps. Characteristics of trapped ions can be investigated at detailed levels within a reasonable simulation time. Space charge effects on the trapping capacities of linear and 3D ion traps, ion cloud shapes, ion motion frequency shift, mass spectrum peak coalescence effects between two ion clouds of close $\mathrm{m} / \mathrm{z}$ are studied with the ion trajectory simulation using GPU.
\end{abstract}

Key words: Space charge effects, Quadrupole ion trap, Ion trajectory simulation, Parallel computing, GPU

\section{Introduction}

A s stand-alone devices for mass analysis, quadrupole ion traps are capable of storing ions and analyzing their massto-charge ratios $(\mathrm{m} / \mathrm{z})[1,2]$, more importantly, performing tandem MS experiments and gas-phase ion chemistry [3-6]. When combined with other mass analyzers, it has elevated the performances of hybrid instruments [7-15]. As one of the most critical factors associated with ion trap performance, space charge effect has significant impacts on ion trap dynamic range, mass discrimination, mass accuracy and mass resolution [16-20]. With higher trapping capacities thus less space charge

Electronic supplementary material The online version of this article (doi:10.1007/s13361-012-0448-1) contains supplementary material, which is available to authorized users.

Correspondence to: Wei Xu; e-mail: weixu@bit.edu.cn effects, the use of linear ion traps has resulted in significant improvements in the performance of commercial ion traps and hybrid mass spectrometers $[8,15,21,22]$. To advance this progress, a fast and effective method to characterize the space charge effects in ion traps is desired, which would greatly facilitate the instrumentation process.

Extensive efforts have been made in experimental characterization of space charge effects in quadrupole ion traps. Its influence on ion cloud spatial distribution and mass shift in 3D quadrupole ion traps have been studied using laser tomography methods [23-25]. The relationships between space charge effects and ion trapping capacity, isolation efficiency, fragmentation efficiency, mass discrimination and MS resolution have also been investigated in linear ion traps $[21,22,26,27]$, as well as in a digital ion trap [28, 29], Fourier transform ion cyclotron resonance (FT-ICR) cells [17, 30-32] and Orbitraps [8, 33-35]. 
In addition to the experimental approaches, theoretical modelings and numeric simulations have also been used as powerful tools in the study of space charge effects. Coulombic force has a nonlinear contribution to the ion motion equation (Mathieu's Equation), and it is difficult to use an analytical solution to comprehensively model space charge effects in an ion trap. With approximations at different levels, several theoretical methods have been proposed [18, 36-42], while frequently they need to be used with assistance from ion trajectory simulations [43-49] to provide meaningful results. The calculation of coulombic force requires excessive amount of computation resources, and it is usually not practical to perform large-scale simulations on personal computers. Methods such as factor repulsion [50] have been used to approximate the space charge effect in the simulation, where the coulombic forces among a small number of ions are amplified by a factor to mimic the effects of large number of ions. With the help of parallel computation techniques, the interactions among 1024 ions in a FT-ICR cell have been simulated [51, 52]. Particle-in-cell approaches have also been introduced to model the coulombic ion-ion interactions and their effect on image charges for relatively large number of ions in FT-ICR cells $[46,53]$ and Orbitrap [35]. The accuracy of this method was claimed to be limited by the ion density and the cell dimension [53]. Recently, a "hybrid" algorithm, which divides the simulation region into an "active region" and the other region, has been integrated with SIMION (Scientific Instrument Services, Inc., NJ, USA) for the space charge simulation of large number of ions [54]. In the "active region", two-body ion-ion interactions were calculated directly; in the other region, ions were grouped as "charge diffused clouds" and ion-cloud Coulomb interactions were calculated. Methods using approximations are effective but dependent on the accuracy of the assumption, which is typically based on the knowledge of the ion distribution and the density. Direct simulation without approximation provides accurate information, especially for new devices or ion trapping conditions; however, the associated computation time can also be the longest.

The recent advances in computing technologies, especially parallel computing technologies, have provided us a great opportunity to overcome the computation limitation for simulations. In this work, parallel computing techniques, especially GPU techniques, have been investigated for simulation of space charge effects involving $10^{5}$ ions using a single desktop computer. GPU has originally been developed for accelerated video decoding while recently been increasingly used for floating point arithmetic computation [55]. GPUs are stream processors, which can operate in parallel by applying one kernel to many elements in a stream at once. With the multi-core hardware configuration, GPUs can be used for parallel computing as a single unit or a cluster, and have been demonstrated to be effective in data analysis, theoretical modeling and numeric simulation $[56$, 57]. The implementation of the GPU method can benefit the simulation methods both with and without approximation by significantly improving the calculation speed. In our study, we use the direct simulation without implementing any approximation for demonstrating the capability of the GPU for numeric simulation. Powered by GPU techniques, an acceleration of about 390 times has been achieved for simulating 3D coulombic ion-ion interactions in comparison with a simulation using a single-core CPU. The space charge effects in quadrupole ion traps have been characterized, which include ion cloud shape, ion trapping capacities for 3D and linear ion traps, ion motional frequency shift, mass shift and MS resolution. Existing popular simulation programs, such as SIMION and ITSIM, potentially can also benefit from this type of technology; however, some modifications in the software are required to make them compatible, as discussed in the Supplemental Information.

\section{Method}

In this study, both 3D and linear ion traps are investigated with ideal geometries and pure quadrupole fields. The 3D ion trap has dimensions of $r_{0}=5 \mathrm{~mm}$ and $z_{0}=3.536 \mathrm{~mm}$ (center to electrodes). The linear ion trap has dimensions of $\mathrm{x}_{0}=\mathrm{y}_{0}=4 \mathrm{~mm}$ (center to electrodes) and an effective length of $30 \mathrm{~mm}$ in the $\mathrm{z}$ direction [22]. Within the effective length, an ideal situation is also assumed for the DC trapping electric field along the z-axis, where the electric field (RF and $\mathrm{DC}$ ) in the $\mathrm{z}$ direction is set at zero. Ions are confined in the $\mathrm{z}$ direction of the linear ion trap by applying mirrored boundary conditions on both ends of the $\mathrm{z}$ direction, which assumes ions never outflow in the $\mathrm{z}$ direction.

Ion trajectory simulation is performed using the ion trajectory simulation program developed in lab using MatLab [58, 59], which solves the Newton's second order differential equation using the 4-th order Runge Kutta integration method. The gas dynamic collision model, Langevin's collision theory, is used to model the ion-neutral collision probabilities. The energy transfer between the ion and neutral molecules during each collision is calculated based on the elastic collision model. Helium is used as the buffer gas (1 mTorr otherwise specified). The simulation time step is set at $50 \mathrm{~ns}$, corresponding to 20 steps in one RF cycle.

The coulombic force $(C F)$ that an ion (labeled as number $x$ ) experiences at each time step is calculated using the following equation:

$$
C F_{x}=\sum_{i=1}^{N} \frac{q_{i} q_{x}}{\varepsilon R_{i x}^{2}} \vec{R}_{i x}
$$

where $N$ is the total number of ions trapped in the ion trap, $q_{\mathrm{i}}$ or $q_{\mathrm{x}}$ denotes the charge on the ion, $\varepsilon$ is the dielectric constant of air, $R_{i x}$ represents the distance between the ion number $x$ and the ion number $i$, and $\vec{R}_{i x}$ is a unit length vector pointing from the ion number $i$ to the ion number $x$. In the current work, an ideal situation is assumed without 
considering the physical geometries of the ion traps, such as the higher-order electric field effects and the image currents or charges induced on the ion trap electrodes. The same simulation method can be used for any real device as long as the resolved field is available, which typically can be obtained using commercial program $[58,59]$. It is a timeconsuming process to calculate Equation (1) for every ion at every time step, especially as the total number of ions increases as shown in Table 1.

To accelerate the simulation process, parallel computing is implemented into the modified ion trajectory simulation program, including both multi-core CPU and GPU techniques. A desktop workstation with $2 \mathrm{CPU}(\operatorname{Intel}(\mathrm{R}) \operatorname{Xeon}(\mathrm{R})$ X5450@3.00GHZ with 2 CPU and 8 core) and a GPU card [NVIDIA C2050 with 448 compute unified device architecture (CUDA) cores) is used to run the simulation. To calculate/predict the position and velocity of an ion at the next time step, knowledge of the forces it experiences at the current position is required, which include the force from the trapping electric field and the coulombic force resulting from the presence of other ions in the trap. Coulombic force calculation is the most time-consuming process at the operating pressure range of ion traps (mTorr range); therefore, the task of calculating coulombic forces for all ions is evenly distributed to different cores at every time step. For example, with 4480 ions in the trap and 448 computing cores in the GPU, the coulombic forces experienced by every 10 ions are calculated by each computing core in the GPU card (Detailed description of the program provided in Supplemental Information).

Efficiency of the ion trajectory simulation is characterized when using different computing units with results shown in Figure S2 and Table 1. Three computing units are investigated for the space charge modeling, single-core CPU, eight-core CPU and 448-core GPU. Table 1 shows the simulation time required to calculate ion motions for a single time step using different computing units, while Figure S2 shows the simulation time required to calculate ion motions within $1 \mathrm{~ms}$, which corresponds to 20000 time steps. When the total number of ions is relatively small (around $10^{3}$ or fewer), the use of parallel computing techniques does not have significant advantages over single-core computation; simulation could be even slower for multi-core computation due to the time required for communications between cores. However, a significant acceleration is obtained for simulation with ions of $10^{4}$ or more, and it is about 8 times and 390 times faster using eight-core CPU and 448-core GPU, respectively. As shown in Figure S2, the simulation time has been reduced from 1.8 year to 1.7 day for a relative realistic simulation case: trajectory simulation of $10^{5}$ ions within $1 \mathrm{~ms}$. As an efficient and economic method, the 448-core GPU is used in this study to characterize the space charge effects in quadrupole ion traps.

\section{Results and Discussion}

Previous studies have shown that ions stored in a 3D ion trap forms an ion cloud that has a Gaussian distribution in each direction of the Cartesian coordinate [25]. Under typical operating conditions (room temperature or higher), an ion oscillates within the ion cloud at its secular frequency. The formation of the ion cloud is a result of the balancing among the forces due to the trapping electric field, the ion-molecule collisions and the coulombic repulsion. The size and shape of the ion cloud have a significant impact on mass analysis performance of an ion trap, such as the sensitivity (associated with the trapping capacity), mass discrimination, dynamic mass range or concentration range, mass accuracy and mass resolution $[15,22]$.

\section{Space Charge Effects on Ion Trapping}

Ions can be generated either internally with ionization methods such as electron impact and photo ionizations [60], or externally with atmosphere pressure ionization methods [61]. The initial distribution of an ion cloud strongly depends on the ion introduction method used in an experiment. Therefore, a cooling period is usually applied to remove the extra kinetic and potential energies from the ion cloud before further manipulations. The shapes and distributions of ion clouds under equilibrium conditions after cooling are studied in this work. In the simulations, a group of ions were placed in the center of the ion trap with a spatial

Table 1. Time Required Calculating Ion Motion for One Time Step in the Ion Trajectory Simulation Using Different Computing Systems: Case A: CPU, Single Core; Case B: CPU, Eight Cores; Case C: GPU

\begin{tabular}{lcccrr}
\hline Ion counts $(\mathrm{k})$ & \multicolumn{1}{c}{ A: CPU } & B: CPU & C: GPU (s) & A/B & A/C \\
\cline { 2 - 5 } & single core (s) & 8 core (s) & & \\
\hline 1 & 0.28078 & 0.740855 & 0.077926 & 0.378995 & 3.603161974 \\
10 & 26.09999 & 3.735379 & 0.163469 & 6.98724 & 159.6632389 \\
100 & 2813.289 & 328.9001 & 7.460955 & 8.553629 & 377.0682238 \\
200 & 11581.02 & 1345.435 & 29.91633 & 8.607641 & 387.1136478 \\
500 & 73725.83 & 8366.219 & 185.7939 & 8.812324 & 396.8151406 \\
1000 & 295318.7 & 33576.14 & 751.8735 & 8.795491 & 392.7770519 \\
\hline
\end{tabular}

CPU: Intel(R) Xeon(R) X5450@3.00GHZ (2 CPU, 8 cores);

GPU: NVIDIA C2050 (448 CUDA cores) 
distribution: Gaussian distributions in the $\mathrm{r}$ and $\mathrm{z}$ directions of the 3D ion trap, while Gaussian distributions in the $\mathrm{x}$ and $\mathrm{y}$ directions and a uniform distribution in the $\mathrm{z}$ direction of the linear ion trap. Initial velocity distributions are Maxwell distributions in all cases. A cooling time of $10 \mathrm{~ms}$ is used to allow the ion cloud to reach its equilibrium state, when the shape and dimensions of the cloud have converged.

The effects of the trapping electric field were characterized by placing $10^{4}$ ions $(\mathrm{m} / \mathrm{z} 500)$ inside the 3D ion trap at different RF voltages $\left(V_{\mathrm{RF}}\right)$, thus at different $q$ values. Figure 1 illustrates the sizes and the shapes of the ion cloud at different $q$ values. The normalized standard deviation (STD) was extracted to depict the size of the ion cloud. From Mathieu's Equation, $q_{\mathrm{z}}$ is two times the value of $q_{\mathrm{r}}$ at a fixed RF voltage for the $3 \mathrm{D}$ ion trap. Due to the linear relationship between the pseudo-potential well depth $(D)$ and the $q$ value $\left(D \sim q V_{\mathrm{RF}}\right)$, the trapping well depth in the $\mathrm{z}$ direction is two times deeper than that in the $\mathrm{r}$ direction at low $q$ regions $(q<0.4)$, which accounts for the elliptical shape of the ion cloud with major axis in the $r$ direction at low $q_{\mathrm{z}}$ values. As the trapping voltage increases, the pseudopotential well depth in the $r$ direction keeps increasing, while the trapping well depth in the $\mathrm{z}$ direction becomes shallower when $q_{z}$ is approaching the boundary of the stability diagram. The ion cloud is squeezed in the $r$ direction and stretched in the $\mathrm{z}$ direction, which results in an elliptical shape with major axis in the $\mathrm{z}$ direction as shown in Figure $1 \mathrm{~b}$. This is a phenomenon not present for linear ion traps, since $q_{\mathrm{x}}$ and $q_{\mathrm{y}}$ are normally identical, and ion clouds will have the same dimension in the $\mathrm{x}$ and $\mathrm{y}$ directions. Without taking the space charge effects into account, ions inside an ion trap will experience the RF trapping field and buffer gas cooling effects and the ion cloud size will be independent of ion number. After cooling, ion thermal energy, which depends on the thermal temperature of the ion trap environment, will balance with the RF trapping potential, and an ion cloud will show similar shape but much smaller dimensions under normal temperature conditions $(<300 \mathrm{~K})$.

In a typical ion trap operation process, an RF of constant amplitude is applied to the ion trap during the ion injection and cooling periods. Ions within a range of $\mathrm{m} / \mathrm{z}$ values can be trapped at the same time. Ions with different $\mathrm{m} / \mathrm{z}$ values are experiencing the same RF voltage but different $q$ values, thus forming ion clouds of different shapes as shown in Figure 2. In Figure 2a, a $400 \mathrm{~V}_{\mathrm{p}-\mathrm{p}} \mathrm{RF}$ voltage is applied on the $3 \mathrm{D}$ ion trap, and $10^{4}$ ions with $\mathrm{m} / \mathrm{z}$ values ranging from 200 to 2000 were placed in the 3D ion trap, respectively. The size of the cloud is dependent on the potential well depth at which the ions of a particular $m / z$ value are trapped. At low $q$ values, heavy ions generally form bigger ion clouds due to the corresponding lower $q$ values. However, the $q_{\mathrm{z}}$ value is twice that of the $q_{\mathrm{r}}$ value, and the ion distribution in the z direction begun to increase as the $\mathrm{m} / \mathrm{z}$ of the trapped ions is reduced and their $q_{z}$ values approach 0.908 as shown in Figure 2a for ions with $\mathrm{m} / \mathrm{z} 200\left(q_{\mathrm{z}}\right.$ 0.78 ). Four types of ions (2500 each) with different $\mathrm{m} / \mathrm{z}$ values (200, 500, 1000 and 2000, respectively) are placed in the 3D ion trap simultaneously with the radius distribution plotted in Figure 2b. This phenomenon is because ions with lower $m / z$ experience stronger RF trapping forces at low $q$ values. This result explains previous experimental observations [23, 24], where light ions were found to form smaller ion clouds in a quadrupole ion trap [62], and confirms the relationship between mass discrimination and space charge effects [15, 63].

Besides the trapping electric fields, the buffer gas cooling effect can counteract the expansion effect due to space (a)

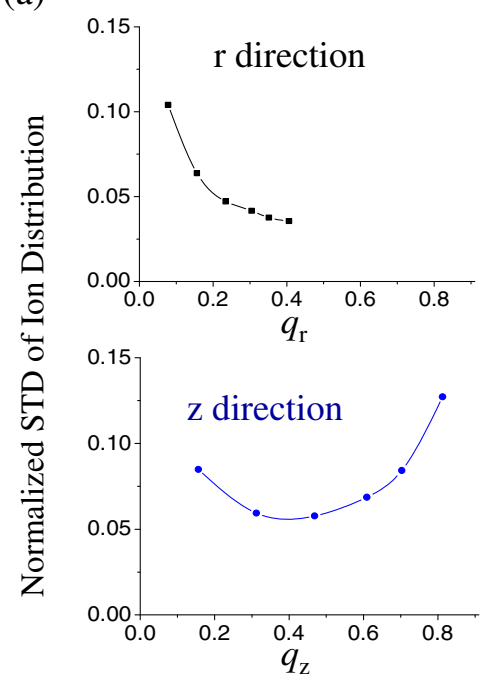

(b)

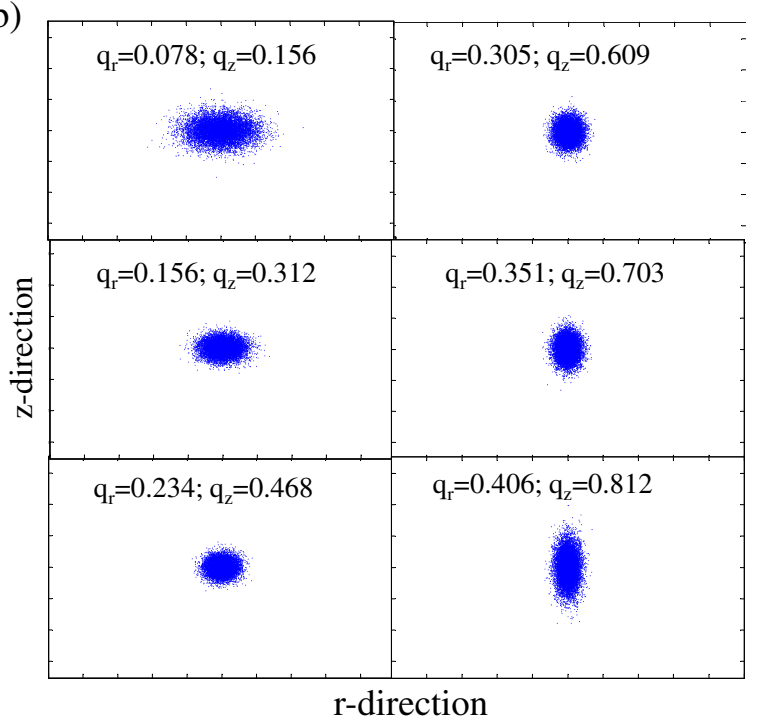

Figure 1. Shapes and sizes of the ion cloud at different RF trapping voltages in an ideal $3 \mathrm{D}$ ion trap. Ion trap dimension: $r_{0}=5 \mathrm{~mm}$, $\mathrm{z}_{0}=3.536 \mathrm{~mm}$. lon cloud: $10^{4}$ ions, $\mathrm{m} / \mathrm{z} 500$ 
(a)

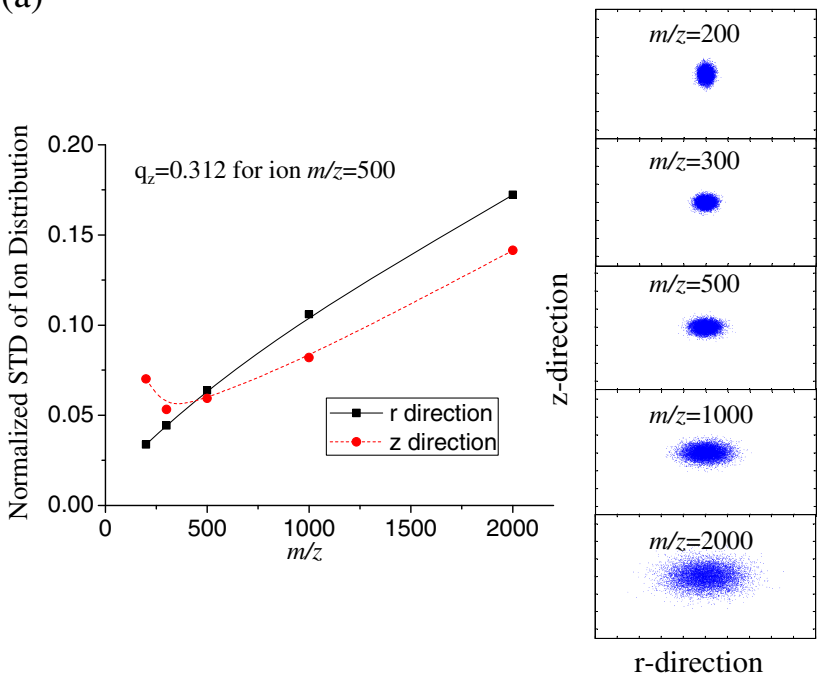

(b)

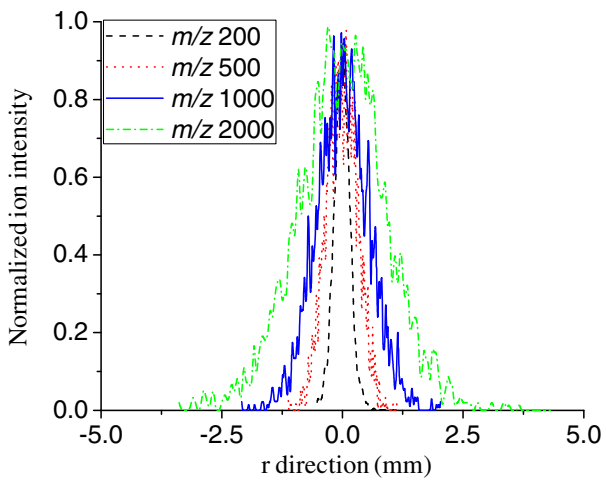

Figure 2. Ion clouds in the 3D ion trap for ions of different $m / z$ values and experiencing the same RF voltage. (a) only ions with the same $\mathrm{m} / \mathrm{z}$ are placed in the ion trap at one time. $10^{4}$ ions in each ion cloud. (b) when four types of ions trapped at the same time. 2500 ions for each type of ions. $V_{\mathrm{RF}}=400 \mathrm{~V}_{\mathrm{p}-\mathrm{p}}$, corresponding to $q_{\mathrm{z}}=0.312$ for ion $\mathrm{m} / \mathrm{z} 500$

charge. The relationship between the ion cloud size (in the $r$ direction of the 3D ion trap) and the buffer gas pressure of helium is shown in Figure 3. In this simulation, $10^{4}$ ions of $\mathrm{m} / \mathrm{z} 500$ are placed in the 3D ion trap with an RF voltage of $400 \mathrm{~V}_{\mathrm{p}-\mathrm{p}}$. As expected, the ion cloud size decreases by increasing the buffer gas pressure from 0.1 to 5 mTorr [6466]. However, not much change is observed when the pressure was increased from 5 to 10 mTorr. Although the reason is still under investigation, interestingly, measurements of the ion trapping efficiency of externally generated ions are also found to be optimal at $\sim 5$ mTorr for linear ion traps $[64,67]$. On the other hand, mass analysis resolution of an ion trap depends not only on the ion cloud size but also the ion motion frequency peak width [65]. Buffer gas can cool the ion cloud but also broaden the ion motion frequency

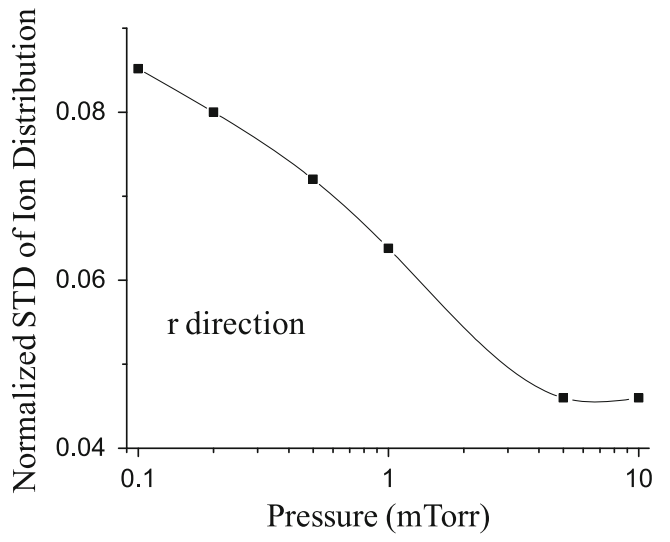

Figure 3. Pressure effect on the size of ion cloud in the 3D ion trap. Ion cloud of $10^{4}$ ions $m / z 500 ; V_{\mathrm{RF}}=400 \mathrm{~V}_{\mathrm{p}-\mathrm{p}}$; helium as the buffer gas peak width. In previous studies, it has been shown that the best resolution can be obtained at $10^{-4} \mathrm{mTorr}$ regime [67].

The trapping capacity of an ion trap relates directly to the space charge effect. It is believed that linear ion traps have much larger trapping capacities when compared with $3 \mathrm{D}$ ion traps. However, there has not been a good way to do a direct comparison experimentally in addition to the simple calculations of the trapping volumes [12, 22]. To address this issue, ion clouds with increased number of ions are simulated and characterized in both the 3D and the linear ion traps. Ions $(\mathrm{m} / \mathrm{z} 500)$ were placed in the ion traps at a $q$ value of $0.156\left(q_{\mathrm{x}}\right.$ for the linear ion trap and $q_{\mathrm{z}}$ for the 3D ion trap). In reality, ion distribution in the $\mathrm{z}$ direction of a linear ion trap is not uniform due to the non-ideal DC electric field distribution close to the end electrodes, and DC end electrode voltage have an effect on the spectral space charge limit by a factor of two [22]. However, an ion distribution in $\mathrm{z}$ direction could be close to a uniform distribution at the center (z direction) of a linear ion trap, when the DC voltages are minimized for optimum ion ejection. For an ideal linear ion trap in this study, a uniform ion distribution in $\mathrm{z}$ direction is assumed (detailed discussions in Supplemental information). Simulation within a segment of the linear ion trap $(20 \mu \mathrm{m})$ would be representative, and a linear relationship is assumed between the trapping capacity of the linear ion trap and that of the segment [22].

As shown in Figure 4, the size of the ion cloud increases in the radical direction for a $3 \mathrm{D}$ ion trap and $\mathrm{x}$ or $\mathrm{y}$ direction for a linear ion trap, as the number of ions increases. Plotted in logarithmic scale, Figure $4 \mathrm{a}$ gives us a direct comparison of the ion trapping capacities of the 3D ion trap with that of the linear ion trap. For instance, when the ion clouds have a normalized STD of 0.123 in both traps, the number of ions 
(a)

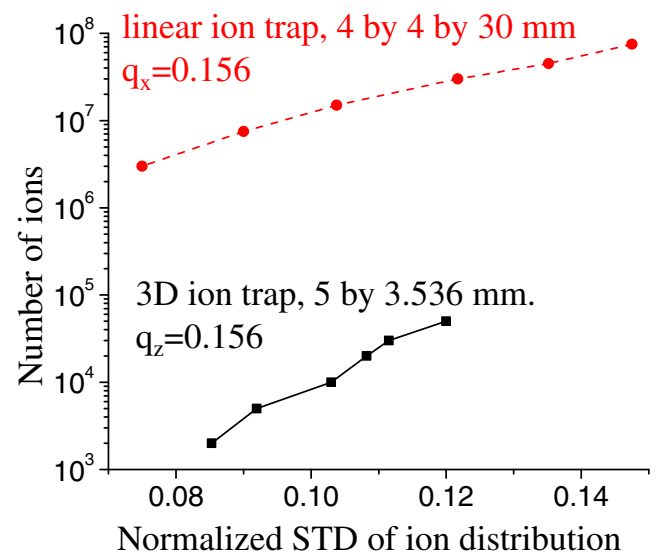

(b)

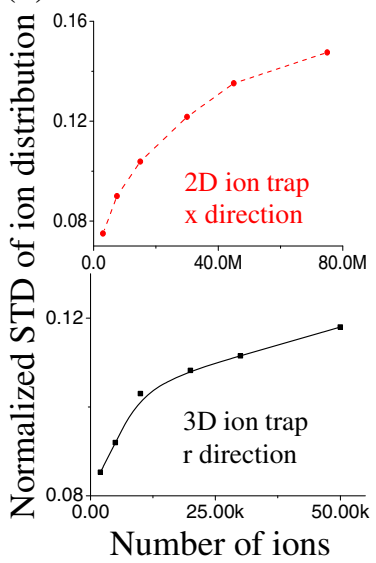

Figure 4. Ion cloud size as a function of number of ions in the 3D and linear ion traps. (a) Log plot. (b) Linear plot. lon $m / z 500$; 3D ion trap: $q_{\mathrm{r}}=0.078, q_{\mathrm{z}}=0.156$; Linear ion trap: $q_{\mathrm{x}}=q_{\mathrm{y}}=0.156$

that trapped in the linear ion trap is about 400 times more than that of the 3D ion trap. This value is about 26 times higher than the value calculated by the trapping volume method [22]. Several reasons could contribute to the difference: 1) in the simulation, the ion cloud has a uniform distribution in the $\mathrm{z}$ direction of the linear ion trap, while Gaussian distribution in the $\mathrm{z}$ direction of the 3D ion trap. However, for a real LIT with electric fields truncated at the ends and the penetration of the trapping DC fields, the real trapping capacity could be lower. 2) The shapes of the ion clouds are different. Cross section (x-y plane) of the ion cloud in the linear ion trap has a round shape, while an elliptical shape in the 3D ion trap at the specific $q_{\mathrm{z}}$ value (0.312). 3) Ion trapping in this work is compared at the same ion cloud radial distribution rather than at a fixed charge density in the trapping volume calculation $[12,22]$. 4) The fixed charge density assumption is not used in this work [12]. As shown later in Figure 4b, it is found that charge density is a function of the total number of ions trapped as

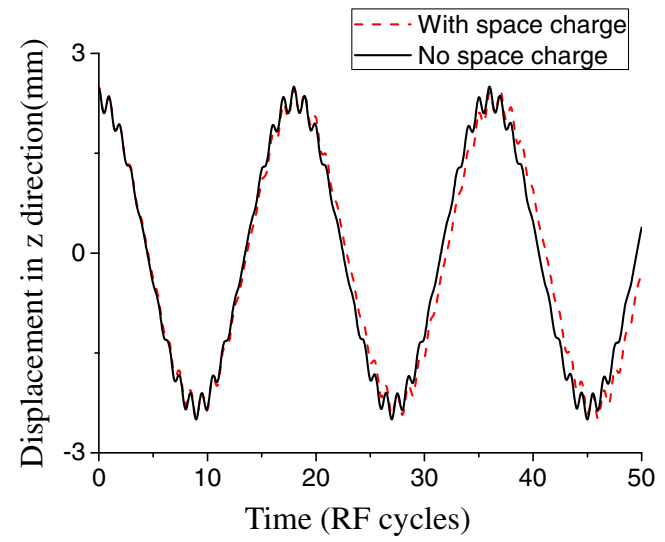

Figure 5. The trajectory of one ion of $\mathrm{m} / \mathrm{z} 500$ with and without $10^{5}$ ions of $\mathrm{m} / \mathrm{z} 600$ trapped simultaneously.. Ion $(\mathrm{m} / \mathrm{z}$ 500 ) initial position $(0,0,2.5 \mathrm{~mm})$; zero initial velocity in all directions. $\mathrm{V}_{\mathrm{RF}}=200 \mathrm{~V}_{\mathrm{p}-\mathrm{p}}$, no ion-molecule collision well as the geometry of the ion trap (linear ion trap vs. 3D ion trap).

Figure $4 \mathrm{~b}$ plots the size of the ion cloud as a function of the number of ions in linear scales. The ion clouds expand rapidly when the STD of the ion cloud size is less than $\sim 0.1$, and expand slower after that. The results indicate that the ion density within the ion cloud increases as the number of ions increases in both traps. A possible reason could be: with less number of ions, ion thermal motion is dominant over the space charge effects, and ion thermal motion has a bigger contribution to the ion cloud size than the space charge

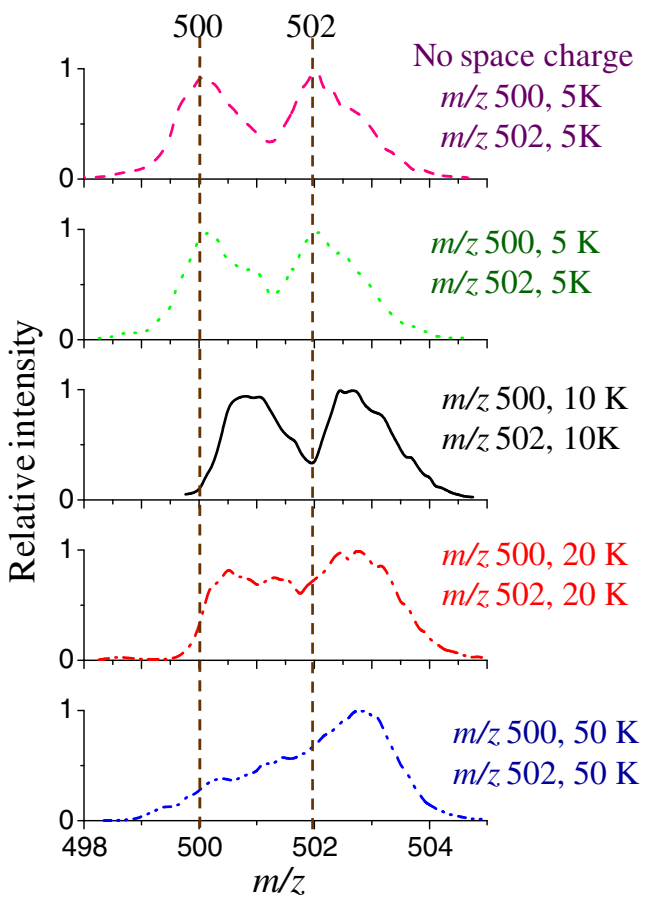

Figure 6. Space charge effect on mass shift and mass resolution, ions of $\mathrm{m} / \mathrm{z} 500$ and 502, placed in the 3D ion trap with Gaussian distributions, boundary ejection, RF scan speed at $10^{5} \mathrm{~V} / \mathrm{s}$ 
effects. As the number of ions increases, space charge effects will be dominant and affect the ion cloud size. Intuitively, if we only put several frogs (low density ions) in a big box (ion trap), they will jump within the box (thermo motion) and occupy a big portion of the box without colliding with each other (Coulomb force). As we put many more frogs (high density ions) in the box, they will still occupy the same box but colliding with each other. On the other hand, as the ion trap dimension scales, while keeping other parameters unchanged ( $R F$ frequency, electric field distribution, etc.), the ion trapping capacity will decrease nonlinearly. Similar phenomenon has been observed experimentally in a 3D ion trap [23].

\section{Space Charge Effects on Ion Trajectory}

Without considering space charge and ion-molecule collisions, ion trajectory inside an RF quadrupole electric field is the summation of a harmonic motion (secular motion) and high-order motions. At low $q$ region $(q<0.4)$, the ion secular motion is dominant, and the ion motion can be approximated as a harmonic oscillator. When considering space charge effects, an ion experiences a position dependant coulombic force, which leads to an effective shift in the ion motion frequency. Figure 5 illustrates this effect, where the trajectories of a specific ion $[\mathrm{m} / \mathrm{z} 500$, initial position $(0,0$, $2.5 \mathrm{~mm})$, initial velocity $(0,0,0)]$ are simulated in two cases: with and without considering coulombic forces. Besides the ion under study, an ion cloud consisted of $10^{5}$ ions $(\mathrm{m} / z 600)$ was also placed in the center of the 3D ion trap with Gaussian spatial distributions. An RF voltage of $200 \mathrm{~V}_{\mathrm{p} \text {-p }}$ is applied on the ion trap. To isolate the space charge effect, ion-molecule collision was ignored in this study. As shown in Figure 5, the space charge effects can effectively lower the ion motion frequency, which induces a mass shift during the mass analysis process, as discussed in the next section. With the presence of an ion cloud of $10^{5}$ ions, the secular frequency of ion $(m / z 500)$ is shifted from $\sim 55.4 \mathrm{kHz}$ to $\sim 54.1 \mathrm{kHz}$.

\section{Space Charge Effects on MS Shift and Resolution}

It is known from experimental studies that ion ejection can be delayed when an excessive amount of ions are trapped in the ion trap, which leads to mass shifts in the MS spectra acquired [16, 68]. It is also believed that MS resolution degradation can also attribute to the space charge effects [22]. A series of simulation has been performed to investigate these effects. Different number of ions with $\mathrm{m} / \mathrm{z}$ 500 and 502 are placed in the 3D ion trap with Gaussian distributions in space. By scanning the RF amplitude at a speed of $10^{5} \mathrm{~V} / \mathrm{s}$, ions are ejected out of the ion trap in the order of their $\mathrm{m} / \mathrm{z}$ values, which is known as ion boundary ejection. As shown in Figure 6, MS shifts and poorer resolutions can be observed by increasing the number of ions. With 5000 or fewer ions of each $\mathrm{m} / \mathrm{z}$ values, the two peaks $(\mathrm{m} / z 500$ and 502) can be resolved with a $\sim 35 \%$ valley with boundary ejection. Mass shift can be clearly observed when the ion numbers are increased to $10^{4}$. The valley between these two peaks was elevated to $\sim 65 \%$ with $2 * 10^{4}$ ions, while $\mathrm{m} / \mathrm{z} 500$ peak merges into the $\mathrm{m} / \mathrm{z} 502$ peak completely when the ion numbers are increased to $5^{*} 10^{4}$. As shown in Figure $4 \mathrm{~b}$ and discussed earlier, ion density will increase faster and the space charge effects will be prominent when the total number of ions is above $10^{4}$ within the 3D ion trap. Interestingly, mass spectra peak shift and coalescence effects happens after the total number of ion is $2 \times 10^{4}$ and above, which agrees with the ion cloud size calculations. This is also in agreement with the findings in previous work, which showed that spectral manifestations of space charge became apparent in number regime of $10 \mathrm{k}-$ $50 \mathrm{k}[15,22]$.

\section{Conclusions}

Parallel computing technologies have been explored for the ion trajectory simulation involving large number of ions. Simulation of space charge effects for ion clouds containing up to $10^{5}$ ions have been achieved on a single desktop computer. The space charge effects on ion cloud shape, ion trapping capacity, ion trajectory, mass accuracy and mass resolution have been investigated. Characterization of both $3 \mathrm{D}$ and linear ion traps can now be studied with simulations including large number of ions. Interestingly, the number of ions trapped in the linear ion trap $\left(x_{0}=y_{0}=4 \mathrm{~mm}\right.$, effective length $30 \mathrm{~mm}$ ) is found to be $\sim 400$ times higher than that of the 3D ion trap $\left(r_{0}=5 \mathrm{~mm}, z_{0}=3.536 \mathrm{~mm}\right)$ under the same $q$ value ( $q_{\mathrm{z}}$ of the $3 \mathrm{D}$ trap $=q_{\mathrm{x}}$ of the linear ion trap $=0.156$ ). This approach provides researchers a rapid, efficient and economic solution to characterize the space charge effects in ion trap instrumentation processes.

\section{Acknowledgements}

This work was supported by National Scientific Instrumentation Grant Program of China (2011YQ09000502 and 2011YQ09000501) and National Science Foundation (0847205-CHE).

\section{References}

1. March, R.E., Todd, J.F.J.: Quadrupole Ion Trap Mass Spectrometry, 2nd edn. John Wiley \& Sons Inc, Hoboken, New Jersey (2005)

2. Dawson, P.H.: Quadrupole Mass Spectrometry and its Applications. Elsevier Scientific Publishing Company, Amsterdam-Oxford-New York (1976)

3. McLuckey, S.A., Goeringer, D.E., Glish, G.L.: Selective Ion Isolation/ Rejection Over a Broad Mass Range in the Quadrupole Ion Trap. J. Am. Soc. Mass Spectrom. 2(1), 11-21 (1991)

4. Jackson, G.P., King, F.L., Goeringer, D.E., Duckworth, D.C.: GasPhase Reactions of $\mathrm{U}+$ and $\mathrm{U} 2+$ with $\mathrm{O} 2$ and $\mathrm{H} 2 \mathrm{O}$ in a Quadrupole Ion Trap. J. Phys. Chem. A 108(11), 2139-2139 (2004)

5. Wu, H.-F., Brodbelt, J.S.: Effects of reactant ion kinetic energy on both endothermic and exothermic ion/molecule reactions in a quadrupole ion trap mass spectrometer. Int. J. Mass Spectrom. Ion Process 124(3), 175-184 (1993) 
6. Xu, W., Charipar, N., Kirleis, M.A., Xia, Y., Ouyang, Z.: Study of Discontinuous Atmospheric Pressure Interfaces for Mass Spectrometry Instrumentation Development. Anal. Chem 82(15), 6584-6592 (2010)

7. Le Blanc, J.C.Y., Hager, J.W., Ilisiu, A.M.P., Hunter, C., Zhong, F., $\mathrm{Chu}$, I.: Unique scanning capabilities of a new hybrid linear ion trap mass spectrometer (Q TRAP) used for high sensitivity proteomics applications. PROTEOMICS 3(6), 859-869 (2003)

8. Makarov, A., Denisov, E., Kholomeev, A., Balschun, W., Lange, O., Strupat, K., Horning, S.: Performance Evaluation of a Hybrid Linear Ion Trap/Orbitrap Mass Spectrometer. Anal. Chem 78(7), 2113-2120 (2006)

9. Parks, B.A., Jiang, L., Thomas, P.M., Wenger, C.D., Roth, M.J., Boyne, M.T., Burke, P.V., Kwast, K.E., Kelleher, N.L.: Top-Down Proteomics on a Chromatographic Time Scale Using Linear Ion Trap Fourier Transform Hybrid Mass Spectrometers. Anal. Chem 79(21), 7984-7991 (2007)

10. Pringle, S.D., Giles, K., Wildgoose, J.L., Williams, J.P., Slade, S.E., Thalassinos, K., Bateman, R.H., Bowers, M.T., Scrivens, J.H.: An investigation of the mobility separation of some peptide and protein ions using a new hybrid quadrupole/travelling wave IMS/oa-ToF instrument. Int. J. Mass Spectrom 261(1), 1-12 (2007)

11. Xia, Y., Wu, J., McLuckey, S.A., Londry, F.A., Hager, J.W.: Mutual storage mode ion/ion reactions in a hybrid linear ion trap. J. Am. Soc. Mass Spectrom. 16(1), 71-81 (2005)

12. Campbell, J.M., Collings, B.A., Douglas, D.J.: A new linear ion trap time-of-flight system with tandem mass spectrometry capabilities. Rapid Comm. Mass Spectrom 12(20), 1463-1474 (1998)

13. Doroshenko, V.M., Cotter, R.J.: A quadrupole ion trap/time-of-flight mass spectrometer with a parabolic reflectron. J. Mass Spectrom 33(4), 305-318 (1998)

14. Syka, J.E.P., Marto, J.A., Bai, D.L., Horning, S., Senko, M.W., Schwartz, J.C., Ueberheide, B., Garcia, B., Busby, S., Muratore, T., Shabanowitz, J., Hunt, D.F.: Novel Linear Quadrupole Ion Trap/FT Mass Spectrometer: Performance Characterization and Use in the Comparative Analysis of Histone H3 Post-translational Modifications. J. Proteome Res 3(3), 621-626 (2004)

15. Douglas, D.J., Frank, A.J., Mao, D.: Linear ion traps in mass spectrometry. Mass Spectrom. Rev 24(1), 1-29 (2005)

16. Cox, K.A., Cleven, C.D., Cooks, R.G.: Mass shifts and local space charge effects observed in the quadrupole ion trap at higher resolution. Int. J. Mass Spectrom. Ion Process 144(1-2), 47-65 (1995)

17. Ledford, E.B., Rempel, D.L., Gross, M.L.: Space charge effects in Fourier transform mass spectrometry. II. Mass calibration. Anal. Chem 56(14), 2744-2748 (1984)

18. Jeffries, J.B., Barlow, S.E., Dunn, G.H.: Theory of space-charge shift of ion cyclotron resonance frequencies. Int. J. Mass Spectrom. Ion Process 54(1-2), 169-187 (1983)

19. Vedel, F., Andre, J.: Influence of space charge on the computed statistical properties of stored ions cooled by a buffer gas in a quadrupole rf trap. Phys. Rev. A 29(4), 2098 (1984)

20. Vedel, F., Andre, J., Vedel, M., Brincourt, G.: Computed energy and spatial statistical properties of stored ions cooled by a buffer gas. Phys. Rev. A 27(5), 2321 (1983)

21. Hager, J.W.: A new linear ion trap mass spectrometer. Rapid Comm. Mass Spectrom 16(6), 512-526 (2002)

22. Schwartz, J.C., Senko, M.W., Syka, J.E.P.: A two-dimensional quadrupole ion trap mass spectrometer. J. Am. Soc. Mass Spectrom. 13(6), 659-669 (2002)

23. Cleven, C.D., Cooks, R.G., Garrett, A.W., Nogar, N.S., Hemberger, P.H.: Radial Distributions and Ejection Times of Molecular Ions in an Ion Trap Mass Spectrometer: A Laser Tomography Study of Effects of Ion Density and Molecular Type. J. Phys. Chem. 100(1), 40-46 (1996)

24. Hemberger, P.H., Nogar, N.S., Williams, J.D., Cooks, R.G., Syka, J.E.P.: Laser photodissociation probe for ion tomography studies in a quadrupole ion-trap mass spectrometer. Chem. Phys. Lett 191(5), 405$410(1992)$

25. Plass, W.R., Gill, L.A., Bui, H.A., Cooks, R.G.: Ion Mobility Measurement by Dc Tomography in an Rf Quadrupole Ion Trap. $J$. Phys. Chem. A 104(21), 5059-5065 (2000)

26. Sugiyama, M.; Hasegawa, H.; Hashimoto, Y. In Dual wire linear ion trap for analyzing large populations of ions, 59th ASMS, THP080: Denver CO (2011).

27. Guna, M., Londry, F.A.: Tandem Ion Trap Design with Enhanced Mass Analysis Capabilities for Large Populations of Ions. Anal. Chem 83(16), 6363-6367 (2011)
28. Ding, L., Sudakov, M., Kumashiro, S.: A simulation study of the digital ion trap mass spectrometer. Int. J. Mass Spectrom 221(2), 117-138 (2002)

29. Ding, L., Sudakov, M., Brancia, F.L., Giles, R., Kumashiro, S.: A digital ion trap mass spectrometer coupled with atmospheric pressure ion sources. J. Mass Spectrom 39(5), 471-484 (2004)

30. Amster, I.J.: Fourier Transform Mass Spectrometry. J. Mass Spectrom 31(12), 1325-1337 (1996)

31. Comisarow, M.B., Marshall, A.G.: Fourier transform ion cyclotron resonance spectroscopy. Chem. Phys. Lett 25(2), 282-283 (1974)

32. Comisarow, M.B., Marshall, A.G.: Frequency-sweep fourier transform ion cyclotron resonance spectroscopy. Chem. Phys. Lett 26(4), 489-490 (1974)

33. Hu, Q., Noll, R.J., Li, H., Makarov, A., Hardman, M., Cooks, R.G.: The Orbitrap: a new mass spectrometer. J. Mass Spectrom 40(4), 430-443 (2005)

34. Makarov, A.: Electrostatic Axially Harmonic Orbital Trapping: A HighPerformance Technique of Mass Analysis. Anal. Chem 72(6), 1156$1162(2000)$

35. Kharchenko, A.; Vladimirov, G.; Heeren, R.; Nikolaev, E., Performance of Orbitrap Mass Analyzer at Various Space Charge and Non-Ideal Field Conditions: Simulation Approach. J. Am. Soc. Mass Spectrom. 23 (5), 977-987 (2012).

36. Grinfeld, D.; Kopaev, I.; Makarov, A.; Monastyrskiy, M. In Spacecharge effects in RF ion storage devices, 59th ASMS, TH 081: Denver, CO (2011).

37. Gross, M.L., Rempel, D.L.: Fourier transform mass spectrometry. Science 226(4672), 261-268 (1984)

38. Nikolaev, E.N., Gorshkov, M.V.: Dynamics of ion motion in an elongated cylindrical cell of an ICR spectrometer and the shape of the signal registered. Int. J. Mass Spectrom. Ion Process 64(2), 115-125 (1985)

39. Chen, S.-P., Comisarow, M.B.: Modelling coulomb effects in Fouriertransform ion cyclotron resonance mass spectrometry by charged disks and charged cylinders. Rapid Comm. Mass Spectrom 6(1), 1-3 (1992)

40. Gorshkov, M.V., Marshall, A.G., Nikolaev, E.N.: Analysis and elimination of systematic errors originating from Coulomb mutual interaction and image charge in fourier transform ion cyclotron resonance precise mass difference measurements. J. Am. Soc. Mass Spectrom. 4(11), 855-868 (1993)

41. Mitchell, D.W., Smith, R.D.: Cyclotron motion of two Coulombically interacting ion clouds with implications to Fourier-transform ion cyclotron resonance mass spectrometry. Phys. Rev. E 52(4), 4366 (1995)

42. Tolmachev, A.V., Udseth, H.R., Smith, R.D.: Charge Capacity Limitations of Radio Frequency Ion Guides in Their Use for Improved Ion Accumulation and Trapping in Mass Spectrometry. Anal. Chem 72 (5), 970-978 (2000)

43. Bui, H.A., Cooks, R.G.: Windows version of the ion trap simulation program ITSIM: a powerful heuristic and predictive tool in ion trap mass spectrometry. J. Mass Spectrom 33(4), 297-304 (1998)

44. Julian, R.K., Nappi, M., Weil, C., Cooks, R.G.: Multiparticle simulation of ion motion in the ion trap mass spectrometer: Resonant and direct current pulse excitation. J. Am. Soc. Mass Spectrom. 6(1), 57-70 (1995)

45. March, R.E., McMahon, A.W., Londry, F.A., Alfred, R.L., Todd, J.F.J., Vedel, F.: Resonance excitation of ions stored in a quadrupole ion trap. Part 1. A simulation study. Int. J. Mass Spectrom. Ion Process 95(2), 119-156 (1989)

46. Mitchell, D.W.: Realistic simulation of the ion cyclotron resonance mass spectrometer using a distributed three-dimensional particle-in-cell code. J. Am. Soc. Mass Spectrom. 10(2), 136-152 (1999)

47. Wu, G., Cooks, R.G., Ouyang, Z., Yu, M., Chappell, W.J., Plass, W.R.: Ion Trajectory Simulation for Electrode Configurations with Arbitrary Geometries. J. Am. Soc. Mass Spectrom. 17(9), 1216-1228 (2006)

48. Lock, C.M., Dyer, E.W.: Simulation of ion trajectories through a high pressure radio frequency only quadrupole collision cell by SIMION 6.0. Rapid Comm. Mass Spectrom 13(5), 422-431 (1999)

49. Austin, D.E., Cruz, D., Blain, M.G.: Simulations of Ion Trapping in a Micrometer-Sized Cylindrical Ion Trap. J. Am. Soc. Mass Spectrom. 17 (3), 430-441 (2006)

50. Wu, G., Noll, R.J., Plass, W.R., Hu, Q., Perry, R.H., Cooks, R.G.: Ion trajectory simulations of axial ac dipolar excitation in the Orbitrap. Int. J. Mass Spectrom 254(1-2), 53-62 (2006) 
51. Miluchihin, N.V., Miura, K., Inoue, M.: Application of a parallel computer to simulation of ion trajectories in an ion cyclotron resonance spectrometer. Rapid Comm. Mass Spectrom 7(11), 966-970 (1993)

52. Nikolaev, E.N., Miluchihin, N.V., Inoue, M.: Evolution of an ion cloud in a Fourier transform ion cyclotron resonance mass spectrometer during signal detection: its influence on spectral line shape and position. International. Int. J. Mass Spectrom. Ion Process 148(3), 145-157 (1995)

53. Nikolaev, E.N., Heeren, R.M.A., Popov, A.M., Pozdneev, A.V., Chingin, K.S.: Realistic modeling of ion cloud motion in a Fourier transform ion cyclotron resonance cell by use of a particle-in-cell approach. Rapid Comm. Mass Spectrom 21(22), 3527 (2007)

54. Saito, K., Reilly, P.T.A., Koizumi, E., Koizumi, H.: A hybrid approach to calculating Coulombic interactions: An effective and efficient method for optimization of simulations of many ions in quadrupole ion storage device with SIMION. Int. J. Mass Spectrom 315, 74-80 (2012)

55. Andrew, T., Extended-precision floating-point numbers for GPU computation. In ACM SIGGRAPH 2006 Research posters, ACM: Boston, Massachusetts (2006).

56. Zhe, F.; Feng, Q.; Arie, K.; Suzanne, Y.-S., GPU Cluster for High Performance Computing. In Proceedings of the 2004 ACM/IEEE conference on Supercomputing, IEEE Computer Society (2004).

57. Jong, H.: Modeling and simulation of genetic regulatory systems: a literature review. Journal of computational biology: a journal of computational molecular cell biology 9(1), 67-103 (2002)

58. Xu, W., Chappell, W.J., Ouyang, Z.: Modeling of ion transient response to dipolar AC excitation in a quadrupole ion trap. Int. J. Mass Spectrom 308(1), 49-55 (2011)

59. Prentice, B.M., Xu, W., Ouyang, Z., McLuckey, S.A.: DC potentials applied to an end-cap electrode of a 3D ion trap for enhanced MSn functionality. Int. J. Mass Spectrom 306(2-3), 114-122 (2011)
60. Karas, M., Hillenkamp, F.: Laser desorption ionization of proteins with molecular masses exceeding 10,000 daltons. Anal. Chem 60(20), 22992301 (1988)

61. Fenn, J.B., Mann, M., Meng, C.K., Wong, S.F., Whitehouse, C.M.: Electrospray ionization for mass spectrometry of large biomolecules. Science 246(4926), 64-71 (1989)

62. Tolmachev, A.V., Udseth, H.R., Smith, R.D.: Radial stratification of ions as a function of mass to charge ratio in collisional cooling radio frequency multipoles used as ion guides or ion traps. Rapid Comm. Mass Spectrom 14(20), 1907-1913 (2000)

63. Belov, M., Nikolaev, E., Anderson, G., Auberry, K., Harkewicz, R., Smith, R.: Electrospray ionization-Fourier transform ion cyclotron mass spectrometry using ion preselection and external accumulation for ultrahigh sensitivity. J. Am. Soc. Mass Spectrom. 12(1), 38-48 (2001)

64. Pekar Second, T., Blethrow, J.D., Schwartz, J.C., Merrihew, G.E., MacCoss, M.J., Swaney, D.L., Russell, J.D., Coon, J.J., Zabrouskov, V.: Dual-Pressure Linear Ion Trap Mass Spectrometer Improving the Analysis of Complex Protein Mixtures. Anal. Chem 81(18), 7757-7765 (2009)

65. Xu, W., Song, Q., Smith, S.A., Chappell, W.J., Ouyang, Z.: Ion Trap Mass Analysis at High Pressure: A Theoretical View. J. Am. Soc. Mass Spectrom. 20(11), 2144-2153 (2009)

66. Song, Q., Xu, W., Smith, S.A., Gao, L., Chappell, W.J., Cooks, R.G., Ouyang, Z.: Ion trap mass analysis at high pressure: an experimental characterization. J. Mass Spectrom 45(1), 26-34 (2009)

67. Schwartz, J. C.; Syka, J. E. P.; Quarmby, S. T. Differential-pressure dual ion trap mass analyzer and methods of use thereof. June, 2008 US Patent 7692142.

68. Plass, W.R., Li, H., Cooks, R.G.: Theory, simulation and measurement of chemical mass shifts in RF quadrupole ion traps. Int. J. Mass Spectrom 228(2-3), 237-267 (2003) 